\title{
Análisis Psicométrico de la Escala adaptada de Procrastinación de Tuckman (APTS)
}

\section{Psychometric Analysis of the adapted Tuckman Procrastination Scale (APTS)}

\author{
Alberto Alegre-Bravo \\ Universidad San Ignacio de Loyola, Lima, Perú \\ ORCID: http://orcid.org/0000-0001-6331-6094 \\ Diego Benavente-Dongo \\ Universidad San Ignacio de Loyola, Lima, Perú \\ ORCID: https://orcid.org/0000-0002-4197-3752
}

Recibido 23-09-19 Revisado 12-12-20 Aprobado 12-05-20 En línea 03-07-20

*Correspondencia

Email: aalegre@usil.edu.pe
Citar como:

Alegre-Bravo, A., \& Benavente-Dondo, D. (2020). Análisis Psicométrico de la Escala adaptada de Procrastinación de Tuckman (APTS). Propósitos y Representaciones, 8(2), e562. doi: http://dx.doi.org/10.20511/pyr2020.v8n2.562 


\section{Resumen}

El objetivo fue analizar las propiedades psicométricas de la escala Adaptada de Procrastinación de Tuckman (ATPS) a partir de su aplicación a 764 estudiantes pertenecientes a una Universidad Privada de Lima metropolitana. Se empleó el análisis factorial exploratorio como evidencia de validez relacionada a la estructura interna de la escala, obteniéndose una estructura unidimensional que subyace a los 15 ítems que la conforman, sin embargo, tres ítems obtuvieron pesos factoriales menores a 0.32 , siendo la escala de 12 ítems la que evidencia mejores índices de ajuste. Los coeficientes de confiabilidad alfa de Cronbach y omega de McDonald resultaron superiores a 0.70 en todos los casos, evidenciándose consistencia interna. Se obtuvo evidencia de validez en relación con otra variable, a través de la correlación entre las puntuaciones en ATPS y el rendimiento académico, siendo negativa y estadísticamente significativa. Se concluye que la ATPS cuenta con propiedades psicométricas adecuadas para seguir con estudios de validación y se recomienda ampliar la muestra con el objetivo de elaborar baremos.

Palabras clave: Procrastinación; Procrastinación académica; Confiabilidad; Validez.

\section{Summary}

The objective was to analyze the psychometric properties of the Adapted Tuckman Procrastination Scale (ATPS) from its application to 764 students belonging to the private university of metropolitan Lima. The exploratory factor analysis was used as evidence of validity related to the internal structure of the scale, obtaining a one-dimensional structure that underlies the 15 items that make it up, however, three items obtained factorial weights less than 0.32 , the scale being 12 items that show better adjustment rates. McDonald's Cronbach and omega reliability coefficients were greater than 0.70 in all cases, showing internal consistency. Evidence of validity was obtained in relation to another variable, through the correlation between ATPS scores and academic performance, being negative and statistically significant. It is concluded that the ATPS has adequate psychometric properties to continue with validation studies and it is recommended to expand the sample with the objective of developing scales.

Keywords: Procrastination; Academic Procrastination; Reliability; Validity.

\section{Introducción}

En el paradigma vigente de la Educación Superior, basado en el desarrollo de competencias, se enfatiza el valorar y reconsiderar las diferencias individuales para promover experiencias educativas que faciliten el logro en los estudiantes del "aprender a aprender" y "aprender a pensar" (López, 2005). Estos dos objetivos se han revelado específicamente; en los últimos años, como orientadores en la formación de los educandos para la adquisición y utilización oportuna de procesos cognitivos, motivacionales y de autorregulación (Vidal, Salas, Fernández \& García, 2016).

A partir de la implantación del Espacio Europeo de Educación Superior se han producido cambios en los modelos educativos y en las metodologías de enseñanza que pretenden favorecer el autoaprendizaje (Gimeno-Sacristán, 2008), dichas transformaciones han devenido en progresivas propuestas e innovaciones en las universidades latinoamericanas y peruanas orientadas al mismo fin. Es así que, la docencia ya no se centra en la enseñanza, si no en el aprendizaje, y el protagonismo pasa del profesor al alumno. Este último, debe ser el artífice de su propio aprendizaje y el profesor abandona el rol de transmisor de conocimientos para convertirse en el dinamizador de este proceso. Asimismo, dejan de ser prioritarios los conocimientos acumulados dándole paso a un enfoque centrado en las capacidades del alumno; es decir sus competencias (Noguera, Martí, Vargas \& Moncayo, 2014). Los alumnos deben convertirse en personas autónomas, las cuales puedan superar partes del proceso de aprendizaje sin la presencia constante del profesor como único proveedor de conocimientos (Díaz-Barriga, 2011). 
Estas finalidades y competencias para desarrollar en el estudiante se encuentran limitadas a pesar de los esfuerzos en mejora continua de las condiciones de enseñanza en la Educación Superior; ya que, se evidencia cotidianamente en los estudiantes la tendencia a aplazar el cumplimiento de sus actividades o tareas asignadas dejándolas para último momento, lo que denominamos procrastinación (Álvarez, 2010).

La procrastinación es la falta o ausencia de autorregulación en el desempeño y una tendencia en el comportamiento a posponer lo que es necesario para alcanzar una meta (Hsin Chun Chu \& Nam Choi, 2005). Sin embargo, cabe resaltar que la postergación no conlleva necesariamente al incumplimiento de la tarea o el alcance de la meta, sino que tiende a lograrse bajo condiciones de elevado estrés. Se expresa como el retraso intencional en un curso de acción previsto, a pesar de la conciencia de los resultados negativos y usualmente su consecuente desempeño insatisfactorio (Steel, 2007). La creencia subyacente en la conducta de procrastinación es que "después es mejor", por eso se la cataloga como el síndrome "para mañana" siendo dos las principales razones para su presencia, el miedo a fallar y la evaluación de la tarea como aversiva (Lay, 1986).

En el ámbito educativo, la procrastinación académica es definida como un defecto en el desempeño en actividades académicas caracterizado por postergar hasta el último minuto actividades que necesitan ser completadas (Wolters, 2003), ya que según Ellis y Knaus (2002), se aplazan las metas académicas hasta el punto óptimo donde el desempeño requerido sea altamente probable.

Así, este tipo de procrastinación es una forma particular de procrastinación, por lo que investigaciones indican que se encuentra asociada con estrategias poco efectivas de aprendizaje, fallas en la comunicación, bajo promedio académico, tareas aburridas o difíciles, hábitos de estudio inadecuados, excusas falsas, plagio, ansiedad, temor a fallar, depresión, pensamientos irracionales, poca autoeficacia y poca autorregulación (Solomon \& Rothblum, 1984; Lay, 1986; Senecal et al., 1995; Ferrari et al., 1995, Alegre, 2013).

La vasta investigación sobre el constructo procrastinación viene demostrando que no es sólo un problema de la gestión o administración del tiempo; también es un proceso que implica una compleja interacción entre componentes cognitivos, afectivo-motivacionales y conductuales. Los efectos de la procrastinación acarrean serias consecuencias, principalmente en el rendimiento académico de los estudiantes universitarios, quienes se desenvuelven académicamente en función a plazos previamente establecidos (Cao, 2012; Chan, 2011; Clariana et al., 2011; Klassen, Krawchunk \& Rajani, 2008; Hsin Chun Chu \& Nam Choi, 2005).

En cuanto a los instrumentos de autoinforme para evaluar la procrastinación académica pueden mencionarse la de Evaluación de la Procrastinación en Estudiantes (Procrastination Assessment Scale for Students, PASS; Salomón \& Rothblum, 1984), el Cuestionario de Procrastinación de exámenes (Test Procrastination Questionnaire, TPQ; Kalechtein, Hocevar, Zimer \& Kalechestein, 1989), el inventario de Procrastinación de Aitken (Aitken, Procrastination Inventory, API, Aitken., 1992), el Inventario de Estado de Procrastinación Académica (Academic Procrastination State Inventory, APSI; Schowenburg, 1992) y la Escala de Procrastinación de Tuckman (Tuckman Procrastination Scale, TPS, Tuckman, 1990).

Esta última es una medida sobre la tendencia a perder el tiempo, postergar o dejar de hacer cosas que deberían estar hechas. Tuckman (1990) define la procrastinación académica como la falta de autorregulación, que se expresa en la tendencia a posponer actividades que podrían estar bajo su propio control. Este inventario, en su versión original, se componía por 72 ítems que evaluaban las tres dimensiones (autodescripción general de la tendencia a postergar tareas, dificultad para hacer cosas displacenteras y tendencia a culpabilizar a otros por la propia desgracia), quedando reducida a 35 ítems distribuidos en dos dimensiones (Tuckman, 1991). 
La ATPS es una medida unidimensional de la procrastinación académica, versión adaptada en Argentina (Furlan, Piemontesi, Illbele \& Sánchez Rosas, 2010) de la Escala de Procrastinación de Tuckman (Tuckman, 1990).

El proceso de adaptación de la Escala de Procrastinación de Tuckman realizado por Furlan et al. (2010), se llevó a cabo a través de un proceso de traducción directa al español de los 35 ítems que conformaban la escala original en inglés, una prueba piloto $(N=5)$ para análisis del proceso de respuesta y la aplicación a 227 estudiantes de la Carrera de Psicología de la Universidad Nacional de Córdova. Sobre las respuestas de la muestra se realizó el análisis de la estructura interna, a través del análisis factorial exploratorio, quedando conformada por 15 ítems, los cuales presentaron cargas factoriales mayores a .40 , agrupados en un solo factor que explicaba un $33.2 \%$ de la varianza y evidenciando una consistencia interna elevada $(\alpha=0.87)$.

Esta misma versión adaptada, fue sometida a un análisis factorial confirmatorio (Furlan, Heredia, Piemontesi \& Tuckman, 2012) para verificar las propiedades psicométricas y determinar la estructura interna de la escala. Los índices de ajustes obtenidos apoyaron un modelo unidimensional $(\mathrm{RMSEA}=0.045, \mathrm{CFI}=0.99, \mathrm{GFI}=0.98, \mathrm{ACI}=\mathrm{CMIN} / \mathrm{DF}=2.655) \mathrm{y}$ una adecuada consistencia interna $(\alpha=0.94)$. Adicionalmente se obtuvieron evidencias de validez, referidas al criterio, a través del análisis de la correlación entre los puntajes en el ATPS, el rendimiento académico y la ansiedad ante exámenes, encontrándose en el primero una correlación negativa y estadísticamente significativa $(r=-0.217, p<0.001)$ y en el segundo una correlación positiva y estadísticamente significativa $(r=0.430, p<0.000)$.

En base a los revisado, en el contexto de Lima no existen evidencias de validez y confiabilidad de la escala Adaptada de Procrastinación de Tuckman (ATPS) que avale su empleo en la evaluación de este constructo en estudiantes universitarios (Dominguez, Villegas \& Centeno, 2014). Considerando la relevancia de este fenómeno en la educación superior y la necesidad de contar con instrumentos que aporten en los procesos de evaluación, diagnóstico e investigación, se plantea como objetivo realizar un análisis psicométrico de la ATPS y así obtener evidencias de bondad psicométrica en nuestro contexto.

Asimismo, la utilidad de este estudio radica en su relevancia teórica y práctica. En el primer caso servirá de base para estudios posteriores centrados en la elaboración de modelos teóricos y con respecto al segundo, permitirá obtener indicadores sobre los cuales intervenir a través de programas de forma preventiva o remedial.

\section{Método}

\section{Diseño}

El estudio constituye una investigación instrumental (Ato, López \& Benavente, 2013), ya que se orienta a analizar y evidenciar las psicométricas de un instrumento de medición psicológico en el contexto limeño.

\section{Participantes}

Se contó con la participación de 764 estudiantes de pregrado regular, con edades comprendidas entre 16 y 33 años $(M=20.42 ; D E=2.56)$, que cursaban de primero a décimo ciclo, de diferentes carreras de una Universidad Privada de Lima Metropolitana. En referencia al género, 55.6\% fueron hombres $(n=425)$ y el $44.4 \%$ mujeres $(n=339)$. El muestreo empleado fue de tipo intencional. 


\section{Instrumentos}

Procrastinación académica: La ATPS es una medida unidimensional de la Procrastinación académica, versión adaptada en Argentina (Furlan, Piemontesi, Illbele \& Sánchez Rosas, 2010). Está conformada por 15 ítems, con codificación directa (Pospongo comenzar con cosas que no me gustan) e inversa (Siempre que hago un plan de acción, lo sigo), siendo el escalamiento tipo Likert de 5 puntos (Nunca me ocurre, Casi nunca me ocurre, A veces me ocurre, Casi siempre me ocurre, Siempre me ocurre), de forma que se puede obtener un puntaje directo a partir de la suma ponderada de las respuesta en los ítems, así, a mayor puntaje en la escala mayor será la cantidad en el rasgo medido.

Rendimiento académico: Se tomó como medida el rendimiento académico promedio acumulado de los estudiantes durante su formación.

\section{Procedimiento}

El ATPS fue aplicado a los estudiantes dentro del Campus Universitario, en los espacios que congregaban a estudiantes entre clases. De forma preliminar se informó a los estudiantes que la evaluación formaba parte de una investigación, siendo su participación voluntaria, indicándose de forma verbal y escrita que sus respuestas serían tratadas con confidencialidad y en estricto anonimato al momento de reportar los resultados.

\section{Estrategias de análisis}

Para obtener evidencia de validez relacionada con la estructura interna de la ATPS, se empleó la técnica de análisis factorial exploratorio (AFE), siguiendo los pasos recomendados por Lloret, Ferreres, Hernández y Tomás (2017) para su implementación en el programa Factor (LorenzoSeva \& Ferrando, 2006). Se considero como input una matriz de correlaciones policóricas, debido a que los ítems son politómicos (tipo Likert) (Ferrando \& Anguino-Carrasco, 2010). El método de extracción utilizado fue Mínimo cuadrados no ponderados (ULS) y la determinación del número de factores a retener fue a partir de la consideración de la combinación de diferentes criterios: el Análisis paralelo (Horn, 1965), el Promedio Mínimo Parcial (MAP) (Verlicer, 1976) y el Gráfico de Sedimentación. Para valorar la estructura factorial obtenida se consideró como criterio de asignación de ítems en el factor valores iguales o mayores a 0.32 (Tabachnick \& Fidell, 2001).

Se analizó la asociación de las puntuaciones de la prueba con otras variables externas como otra fuente de validación, así se calculó la correlación entre los puntajes obtenidos en APTS y el promedio ponderado a través del estadístico paramétrico de Pearson $(r)$, siendo interpretada la magnitud del coeficiente obtenido considerando los criterios de $0.10,0.30$ y 0.50 de Cohen (1988) como pequeño, mediano y grande, respectivamente.

La confiabilidad de las puntuaciones se obtuvo a través del método de consistencia interna, calculándose los coeficientes alfa de Cronbach $(\alpha)$ y omega de McDonald $(\omega)$.

Cabe resaltar, con respecto al procesamiento de los datos que, se elaboró una base de datos previa en Microsoft Excel donde se registraron las respuestas codificadas de los participantes para todos los campos de información obtenidos. Para el análisis factorial exploratorio se usó el programa FACTOR versión 10.5.03 (Lorenzo-Seva \& Ferrando, 2007) y para el cálculo del coeficiente alfa y las correlaciones se empleó el programa JASP versión 0.8.1.2 (JASP Team, 2017). 


\section{Resultados}

\section{Análisis preliminar de ítems}

En la tabla 1 se puede apreciar el análisis descriptivo, donde la media de los reactivos oscila entre 2.18 (ítem 6) y 3.35 (ítem 12), en cuanto a la dispersión, el ítem 4 posee la baja $(D E=0.84)$ y el ítem 13 la más alta $(D E=1.08)$. Los índices de asimetría $\left(g^{l}\right)$ y curtosis $\left(g^{2}\right)$ evidencian que estos los reactivos presentan valores que se encuentran dentro del rango de $+/-1$ indicando una distribución normal univariada (Ferrando \& Anguino-Carrasco, 2010), siendo en todos los casos aceptables.

Tabla 1.

Análisis descriptivo de ítems

\begin{tabular}{lllll}
\hline Ítem & $M$ & $D E$ & $g^{I}$ & $g^{2}$ \\
\hline Atps1 & 2.63 & 0.84 & 0.18 & -0.01 \\
Atps2 & 2.86 & 0.86 & 0.13 & 0.02 \\
Atps3 & 2.55 & 0.96 & 0.30 & -0.17 \\
Atps4 & 2.68 & 0.98 & 0.28 & -0.20 \\
Atps5 & 2.92 & 0.89 & 0.03 & -0.32 \\
Atps6 & 2.18 & 0.97 & 0.54 & -0.28 \\
Atps7 & 2.81 & 0.96 & 0.05 & -0.55 \\
Atps8 & 2.58 & 0.94 & 0.33 & -0.24 \\
Atps9 & 2.63 & 0.88 & 0.25 & 0.07 \\
Atps10 & 2.59 & 0.96 & 0.28 & -0.16 \\
Atps11 & 2.60 & 1.03 & 0.32 & -0.48 \\
Atps12 & 3.35 & 1.06 & -0.22 & -0.52 \\
Atps13 & 2.60 & 1.08 & 0.36 & -0.47 \\
Atps14 & 2.93 & 0.88 & 0.05 & -0.16 \\
Atps15 & 2.80 & 0.90 & 0.22 & 0.00 \\
\hline
\end{tabular}

Para el análisis de homogeneidad del test, se realizaron las sucesivas correlaciones entre cada ítem y la puntuación total de la escala, no considerando el mismo ítem (Elosua, 2003). Así, se identificó que el ítem $12\left(r_{i t}=0.11\right)$ presentó una correlación menor a $0.20(\mathrm{Kline}, 1998)$ (Tabla 2). 
Tabla 2.

Índices de homogeneidad.

\begin{tabular}{ll}
\hline Ítem & $r_{i t}$ \\
\hline Atps1 & 0.48 \\
Atps2 & 0.43 \\
Atps3 & 0.51 \\
Atps4 & 0.50 \\
Atps5 & 0.30 \\
Atps6 & 0.44 \\
Atps7 & 0.28 \\
Atps8 & 0.48 \\
Atps9 & 0.48 \\
Atps10 & 0.55 \\
Atps11 & 0.39 \\
Atps12 & $\mathbf{0 . 1 1}$ \\
Atps13 & 0.44 \\
Atps14 & 0.28 \\
Atps15 & 0.47 \\
\hline
\end{tabular}

Nota: Correlaciones menores a 0.20 en negrita.

\section{Análisis factorial exploratorio}

Para aportar evidencias de validez vinculadas a la estructura interna de la APTS, se llevó a cabo un análisis factorial exploratorio (AFE) sobre los 15 ítems que conforman el instrumento.

Se analizaron las condiciones previas para aplicar el AFE, así se obtuvo una medida de adecuación muestral de Kaiser-Meyer-Olkin (KMO) de .889, lo cual indica que tiene un idóneo potencial explicativo. El Test de esfericidad de Bartlett fue significativo $\left(\chi^{2}=2330.1 ; p=.000\right)$ y se calculó la matriz de correlaciones policóricas, la cual presentó resultados significativos. Todos estos valores evidencian la pertinencia de llevar a cabo el AFE (Abad, Olea, Ponsoda \& García, 2012).

Se empleó como método de extracción el de Mínimos Cuadrados no Ponderados (ULS) y se determinó el número de factores a retener mediante el análisis paralelo de Horn (1965). Además, siguiendo los lineamientos propuestos por Lloret-Segura, Ferreres-Traver, HernándezBaéza y Tomás-Marco (2014) se consideraron otros métodos como MAP (Verlicer, 1976) y el gráfico de sedimentación (Figura 1), sugiriéndose en los tres casos la extracción de un factor. 


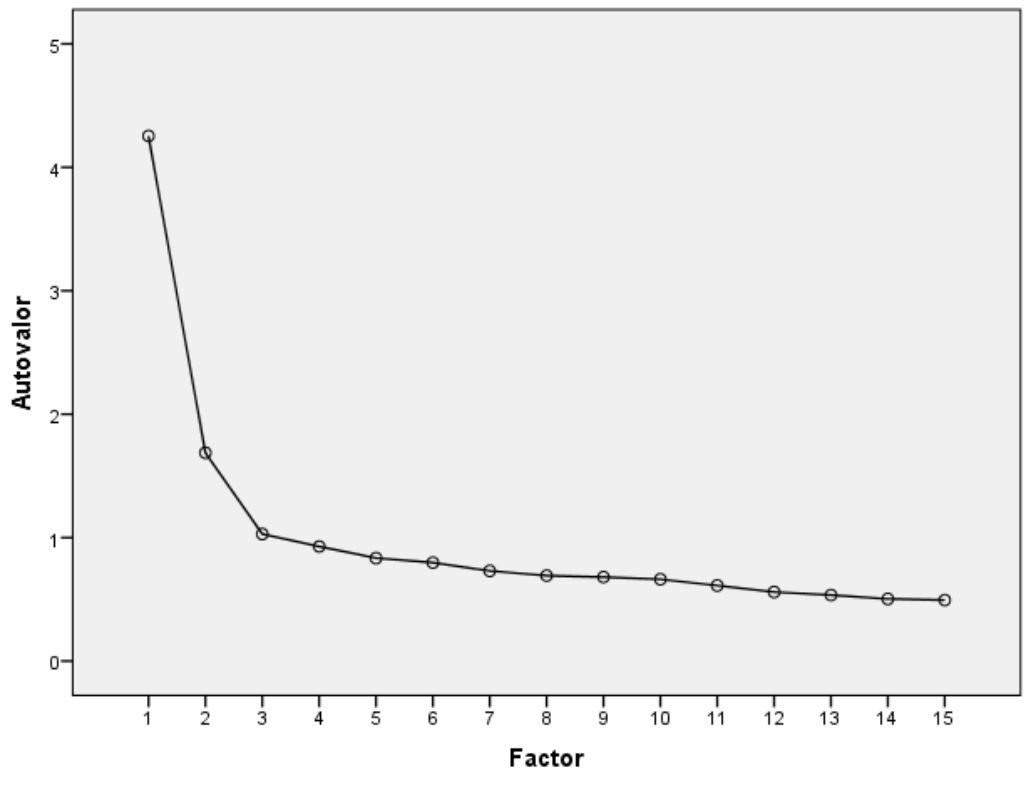

Figura 1. Gráfico de sedimentación

Como resultado se indica una solución unifactorial, la cual explica el 33.35\% de la varianza total, coincidiendo con la versión argentina (Furlan et al., 2012); sin embargo, los ítems 5,7 y 12 no obtuvieron pesos factoriales iguales o mayores a 0.32 (Tabachnick y Fidell, 2001) para considerar su asignación en el factor (Tabla 3).

Tabla 3.

Matriz factorial con 15 ítems

\begin{tabular}{lll}
\hline Ítem & Carga Factorial & Comunalidad \\
\hline Atps1 & 0.614 & 0.377 \\
Atps2 & 0.512 & 0.262 \\
Atps3 & 0.674 & 0.455 \\
Atps4 & 0.610 & 0.372 \\
Atps5 & $\mathbf{0 . 2 5 1}$ & 0.063 \\
Atps6 & 0.591 & 0.349 \\
Atps7 & $\mathbf{0 . 2 6 1}$ & 0.068 \\
Atps8 & 0.653 & 0.426 \\
Atps9 & 0.634 & 0.402 \\
Atps10 & 0.691 & 0.478 \\
Atps11 & 0.404 & 0.164 \\
Atps12 & $\mathbf{0 . 2 7 0}$ & 0.073 \\
Atps13 & 0.563 & 0.317 \\
Atps14 & 0.395 & 0.156 \\
Atps15 & 0.631 & 0.398 \\
\hline Varianza explicada & $33.35 \%$ \\
\hline
\end{tabular}

Determinante $=0.0033$

Medida de adecuación muestral de Kaiser-Meyer-Olkin $=.889$

Test de esfericidad de Bartlett $=2565.6$ g.1. $=105 p=.00001$

Nota: Pesos factoriales menores a 0.32 en negrita. 
Se reanalizó excluyendo los reactivos cuyas cargas factoriales fueron menores a 0.32 (ítems, 5, 7 y 12) en el primer análisis, obteniéndose así una estructura unifactorial que explicaba el $39.85 \%$ de la varianza total y las saturaciones de los reactivos oscilaron entre 0.387 (ítem 14) y 0.669 (ítem 3) (Tabla 4)

Tabla 4.

Matriz factorial con 12 ítems

\begin{tabular}{lll}
\hline Ítem & Carga Factorial & Comunalidad \\
\hline Atps1 & 0.613 & 0.376 \\
Atps2 & 0.513 & 0.264 \\
Atps3 & 0.669 & 0.447 \\
Atps4 & 0.612 & 0.375 \\
Atps6 & 0.600 & 0.360 \\
Atps8 & 0.666 & 0.443 \\
Atps9 & 0.644 & 0.415 \\
Atps10 & 0.682 & 0.465 \\
Atps11 & 0.397 & 0.158 \\
Atps13 & 0.554 & 0.307 \\
Atps14 & 0.387 & 0.150 \\
Atps15 & 0.632 & 0.400 \\
\hline \multicolumn{2}{l}{ Varianza explicada } & $39.85 \%$ \\
\hline Determinante $=0.0056$ & \\
Medida de adecuación muestral de Kaiser-Meyer-Olkin $=.897$ \\
Test de esfericidad de Bartlett = 2189.8.6 g.l. = 66 $p=.00001$ \\
\hline
\end{tabular}

En la tabla 5 se presentan los índices de bondad de ajuste para el AFE del factor extraído, así, para el modelo de 15 ítems se observa que el Error de aproximación de la media cuadrática (RMSEA) muestra un valor de 0.073 (>0.06), el índice de ajuste comparativo (CFI) evidencia un valor de $0.947(<0.95)$ y el índice de bondad de ajuste (GFI) muestra un valor de $0.962(>.95)$. En el caso del modelo para 12 ítems se muestra un RMSEA $=0.056(<0.60), \mathrm{CFI}=0.979(>0.95)$ y $\mathrm{GFI}=0.986(>0.95)$. Siendo, entonces, este último el que evidencia un mejor ajuste.

Tabla 5.

Índices de bondad de ajuste

\begin{tabular}{lllllll}
\hline & Modelo & $X^{2}$ & $g l$ & CFI & GFI & RMSEA \\
\hline ATPS & 15 ítems & 460.151 & 90 & 0.947 & 0.962 & 0.073 \\
& 12 ítems & 184.127 & 54 & 0.979 & 0.986 & 0.056 \\
\hline
\end{tabular}

\section{Validez en relación con otras variables.}

Para evidenciar la validez en relación con otras variables, se correlacionaron las puntuaciones en ATPS con el promedio acumulado de calificaciones obtenidas. A partir del análisis se obtiene que existe relación negativa y estadísticamente significativa $(r=-0.140, p<.001)$, siendo el tamaño del efecto pequeño (Cohen, 1988) (Tabla 6). 


\section{Tabla 6.}

Correlaciones con el rendimiento académico

\begin{tabular}{llll} 
& Rendimiento académico & Procrastinación \\
\hline Rendimiento académico & - & & \\
Procrastinación & -0.140 & $* * *$ & - \\
\hline Nota: $* * * p<.001$ & & &
\end{tabular}

\section{Confiabilidad.}

La confiabilidad de escala se estimó mediante el cálculo de los coeficientes alfa de Cronbach $(\alpha)$ y Omega de McDonald $(\omega)$. Considerando los 15 ítems que componen la escala original se obtuvo valores alfa de Cronbach y Omega de McDonald de 0.845 y 0.848 , respectivamente. En referencia a la estimación de confiabilidad considerando 12 ítems se evidencia un alfa de Cronbach de 0.859 y un coeficiente omega de 0.860 (Tabla 7).

Tabla 7

Confiabilidad de escala

\begin{tabular}{lllllll}
\hline & $N^{\circ}$ de items & $M$ & $D E$ & Rango $r_{i t}$ & $\alpha$ & $\omega$ \\
\hline ATSP & 15 & 2.71 & 0.498 & $0.185,0.589$ & 0.845 & 0.848 \\
& $12^{\mathrm{a}}$ & 2.64 & 0.554 & $0.333,0.581$ & 0.859 & 0.860 \\
\hline
\end{tabular}

Nota. ${ }^{\text {a }}$ Items 5,7 y 12 removidos.

\section{Discusión}

El objetivo del presente estudio fue obtener evidencia de las propiedades psicométricas, tanto validez como confiabilidad de la escala APTS en estudiantes universitarios de Lima Metropolitana.

Para la consecución de dicho objetivo se inició con el análisis descriptivo de ítems, donde se evidenció normalidad univariada; sin embargo, la correlación de cada uno de estos con el puntaje total, como indicador de la homogeneidad, permitió identificar que el ítem 12 presentó un valor menor a 0.20 (Kline, 1998).

En referencia a la evidencia de validez relacionada a la estructura interna, a partir del análisis factorial exploratorio (AFE), se obtuvo una estructura unifactorial, lo cual coincide con lo reportado por los autores de la ATPS (Furlan et al., 2012). El único factor extraído fue sugerido a partir de la consideración de distintos métodos (Análisis paralelo, MAP y Gráfico de sedimentación) lo cual es recomendado para garantizar la idoneidad de factores a retener (LloretSegura et al., 2014); sin embargo, en la matriz factorial resultante se evidenció que tres ítems (5, 7 y 12) obtuvieron pesos factoriales menores a 0.32 . Por ello, se reanalizó factorialmente la escala excluyendo dichos reactivos; a partir de ello se obtuvieron índices de ajuste comparativamente superiores a la versión de 15 ítems. Los 12 ítems que conforman la escala propuesta constituyen indicadores de procrastinación que son identificados en los modelos teóricos de la procrastinación académica (Garzón \& Gil, 2017), es decir la tendencia a posponer responsabilidades (tareas o trabajos) de manera intencionada (Tuckman, 2003) y que evidencian un fallo autorregulatorio (Steel, 2007). En función a lo anteriormente mencionado, se puede colegir que la solución 
obtenida es adecuada, ya que cumple con los criterios de estructura simple, desarrollo positivo y facilidad de interpretación (Domínguez, Villegas, Sotelo \& Sotelo, 2012).

El empleo de un análisis factorial exploratorio y no confirmatorio en este estudio instrumental, se justifica por dos razones: primero, es difícil especificar en ciencias sociales, el valor de las correlaciones con cada factor y segundo, porque no se cuenta con información previa acerca del comportamiento de los ítems y la estructura interna en el contexto peruano, siendo el análisis factorial confirmatorio una técnica que supone un conjunto de supuestos previos (Dominguez, Villegas, Yauri, Mattos \& Ramírez, 2012).

En relación con el rendimiento académico, la correlación entre el puntaje total en ATPS con el promedio ponderado acumulado fue negativa y estadísticamente significativa, con un tamaño del efecto pequeño, al igual a lo obtenido por Furlan et al. (2012); indicando así que la procrastinación académica contribuye a un bajo rendimiento. Este hallazgo se encuentra en correspondencia a lo reportado en las revisiones meta-analíticas del efecto de esta variable sobre el rendimiento académico (Steel, 2007; Rozenthal \& Carlbring, 2014; Kim \& Seo, 2015) en el sentido que la postergación del inicio de las actividades asignadas en los cursos, y la falta de regulación de las conductas propias de estudio conllevan a un bajo desempeño y desarrollo de productos de aprendizaje de baja calidad. Cabe además considerar que el impacto que la procrastinación académica tiene sobre el rendimiento está mediado por otras variables psicológicas y académicas, tales como el valor de la tarea, la duración del curso, el estrés, la autoeficacia, etc. (Garzón \& Gil, 2017).

En cuanto a la confiabilidad del factor extraído, se obtuvo un alfa de Cronbach de 0.845 y un Omega de McDonald de 0.848 para la solución factorial de 15 ítems y para la versión de 12 ítems, coeficientes alfa de Cronbach y omega, de 0.859 y 0.960 , respectivamente. En ambos casos los valores obtenidos son superiores a 0.70 , indicando que se encuentran por encima del nivel considerado adecuado (Campos-Arias \& Oviedo, 2008).

En términos de las limitaciones del estudio, se puede mencionar que los participantes provienen de una Universidad Privada de Lima, por ello se sugiere otros estudios de validación que recolecte datos del ATPS en estudiantes de diversas universidades que incluyan instituciones privadas y públicas de Lima.

Los resultados obtenidos permiten concluir que la ATPS aplicada en estudiantes universitarios de Lima cuenta con propiedades psicométricas sólidas, pues lo reportado concuerda con la estructura unidimensional propuesta por los autores en estudiantes argentinos (Furlan et al, 2012), lo cual evidencia su aplicabilidad y pertinencia más allá del nivel local. Finalmente, el presente estudio pretende aportar a la evidencia empírica a través de la validación de instrumentos a nivel transcultural, dada la relevancia del constructo.

\section{Referencias}

Abad, F., Olea, J., Ponsoda, V., \& García, C. (2012). Medición en ciencias sociales y de la salud. Madrid: Editorial Síntesis.

Alegre, A. (2013). Autoeficacia y procrastinación académica en estudiantes universitarios de Lima Metropolitana. Propósitos y Representaciones. 2(1), 57-82. doi: http://dx.doi.org/10.20511/pyr2013.v1n2.29

Álvarez, O. (2010). Procrastinación general y académica en una muestra de estudiantes de secundaria de Lima metropolitana. Persona, 13, 159-177.

Ato, M., López, J., \& Benavente, A. (2013). Un sistema de clasificación de los diseños de investigación en psicología. Anales de psicología, 29(3), 1038-1059. Doi: http://dx.doi.org/10.6018/analesps.29.3.178511

Campos-Arias, A., \& Oviedo, H. (2008). Propiedades psicométricas de una escala: la consistencia interna. Revista de Salud Pública, 10(5), 831-839. 
Cao, L. (2012). Differences in procrastination and motivation between undergraduate and graduate students. Journal of the Scholarship of Teaching and Learning, 12(2), 39-64.

Chan, A. (2011). Procrastinación académica como predictor en el rendimiento académico en jóvenes de educación superior. Revista Temática Psicológica, 7(1), 53-62.

Clariana, M., Cladellas, R., Badía, M., \& Gotzens, C. (2011). La influencia del género en variables de la personalidad que condicionan el aprendizaje: inteligencia emocional y procrastinación académica. REIFOP, 14(3), 87-96.

Díaz-Barriga, A. (2011). Competencias en educación. Corrientes y pensamiento e implicancias para el currículo y el trabajo en el aula. Revista Iberoamericana de Educación Superior, 2(5), 3-24.

Domínguez, S., Villegas, G., Yauri, C., Mattos, E., \& Ramírez, F. (2012). Propiedades psicométricas de una escala de autoeficacia para situaciones académicas en estudiantes universitarios peruanos. Revista de psicología, 2, 29-39.

Domínguez, S., Villegas, G., \& Centeno, Sh. (2014). Procrastinación académica: Validación de una escala en una muestra de estudiantes de una universidad privada. LIBERABIT, 20(2), 293-304.

Dominguez, S., Villegas, G., Sotelo, N., \& Sotelo, L. (2012). Revisión psicométrica del inventario de Ansiedad Estado-Rasgo (IDARE) en una muestra de universitarios de Lima metropolitana. Revista Peruana de Psicología de Trabajo Social, 1(1), 45-54.

Ellis, A., \& Knaus, W. (2002). Overcoming procrastination. New York: INew American Library. Elousa, P. (2003). Sobre la validez de los tests. Psicothema, 15(2). 315-321.

Ferrando, P. J., \& Anguino-Carrasco, C. (2010). El análisis factorial como técnica de investigación en psicología. Papeles del Psicólogo, 31(1), 18 - 33.

Ferrari, J., Johnson, J., \& Mc Conwn, W. (1995). Procrastination and task avoidance. New York: Plenum Press.

Furlan, L., Heredia, E., Piemontesi, S., \& Tuckman, B. (2012). Análisis factorial confirmatorio de la adaptación argentina de la escala de procrastinación de Tuckman (ATPS). Perspectivas en Psicología: Revista de Psicología y Ciencias Afines, 9(3), 142-149.

Furlan, L., Heredia, D., Piemontesi, S., Illbele, A., \& Sanchez, J. (2010). Adaptación de la escala de procrastinación de Tuckman para estudiantes universitarios. II Congreso Internacional de Investigación y Práctica Profesional en Psicología XVII Jornadas de Investigación Sexto Encuentro de Investigadores en Psicología del MERCOSUR. Facultad de Psicología - Universidad de Buenos Aires, Buenos Aires.

Garzón, A., \& Gil, J. (2017). El papel de la procrastinación académica como factor en la deserción universitaria. Revista Complutense de educación, 28(1), 307-324.

Gimeno-Sacristán, J. (2008). Educar por competencias, ¿qué hay de nuevo? Madrid: Morata.

Horn, J. (1965). A rationale and test for the number of factors in factor analysis. Psychometrika, 30, 179-185.

Hsin Chun Chu, A., \& Nam Choi, J. (2005). Rethinking procrastination: Positive effects of "active" procrastination behavior on attitudes and performance. The Journal of Social Psychology, 145(3), 245-264.

Hu, L., \& Bentler, P. (1998). Fit indices in covariance structure modeling: Sensitvity to underparameterized model misspecification. Psychological Methods, 3(4), 424-453.

JASP Team (2017). JASP (Version 0.8.1.2) [Computer software].

Kim, K. R., \& Seo, E. H. (2015). The relationship between procrastination and academic performance: A meta-analysis. Personality and Individual Differences, 82, 26-33. doi: http://dx.doi.org/10.1016/j.paid.2015.02.038

Klassen, R., Krawchunk, L. \& Rajani, S. (2008). Academic procrastination of undergraduates: Low self-efficacy to self-regulate predicts higher levels of procrastination. Contemporary Educational Psychology, 33, 915-931.

Kline, P. (1998). The new psychometrics: science, psychology and measurement. London: Routhledge.

Lay, C. (1986). At least my research article on procrastination. Journal of Research in Personality, 20, 474-495. 
Lloret-Segura, S., Ferreres-Traver, A., Hernández.Baeza, A., \& Tomás-Marco, I. (2014). El análisis factorial exploratorio de los ítems: una guía práctica, revisada y actualizada. Anales de psicología, 30(3), 1151-1169. doi: http://dx.doi.org/10.6018/analesps.30.3.199361

López, F. (2005). Posibles escenarios de la educación superior. Horizontes, 23(109), 140-165.

Lorenzo-Seva, U., \& Ferrando, P (2007). FACTOR (Version 10.5.03) [Computer software]. Tarragona: University Rovira i Virigili.

Lorenzo-Seva, U., \& Ferrando, P. (2006). FACTOR: A computer program to fit the exploratory factor analysis model. Behavioral Research Methods, Instruments and Computers, 38(1), 88-91.

Muñiz, J. (1994). Teoría clásica de los test. Madrid: Ediciones Pirámide.

Noguera, J., Martí, M., Vargas, O., \& Moncayo, J. (2014). Reflexión sobre los discursos en educación superior, una mirada desde la psicología social crítica. Revista de la educación superior, 43(172), 33-55.

Pérez, E., \& Medrano, L. (2010). Análisis Factorial Exploratorio: Bases Conceptuales y Metodológicas. Revista de Argentina de Ciencias del Comportamiento, 2(1), 58-66.

Rozenthal, A. \& Carlbring, P. (2014). Understanding and Treating Procrastination: A review of a Common Self-Regulatory Failure. Psychology, 5(3), 1488-1502.

Solomon, L. J., \& Rothblum, E. D. (1984). Academic procrastination: Frequency and cognitivebehavioral correlates. Journal of counseling psychology, 31(4), 503.

Senécal, C., Koestner, R. \& Vallerand, R. (1995). Self-Regulation and Academic Procrastination. The Journal of Social Psychology. 135(5), 607-619.

Steel, P. (2007). The nature of procrastination: A meta-analytic and theoretical review of quintessential self-regulatory failure. Psychological Bulletin, 133, 65-94.

Tabachnick, B., \& Fidell, L. (2001). Using multivariate statistics. New York: Harper \& Row.

Tuckman, B.W. (1990). Group versus goal-setting effects on the self-regulated performance of students differing in self-efficacy. Journal of Experimental Education, 58, 291-298.

Tuckman, B. (1991). The development and concurrent validity of the Procrastination Scale. Educational and Psychological Measurement, 51, 473-483.

Tuckman, B.W. (2003). The effect of learning and motivation strategies training on college students' achievement. Journal of College Student Development, 44(3), 430-437.

Verlicer, W.F. (1976). Determining the number of components from the matrix of partial correlations. Psychometrika, 41, 321-327.

Vidal, M.J., Salas, R.S., Oliva, B., \& García, A.L. (2016). Educación basada en competencias. Educación Médica Superior, 30(1), 1-13.

Wolters, C. (2003). Understanding procrastination from a self-regulate learning perspective. Journal of Educational Psychology, 95, 179-187. 


\section{ESCALA DE ATPS}

Sexo: (Hombre) (Mujer)

Edad: ............ años

\section{Instrucciones:}

A continuación, responde con sinceridad a cada una de las afirmaciones abajo enunciadas marcando con un aspa (X) en la alternativa que mejor describa tu experiencia cotidiana como estudiante.

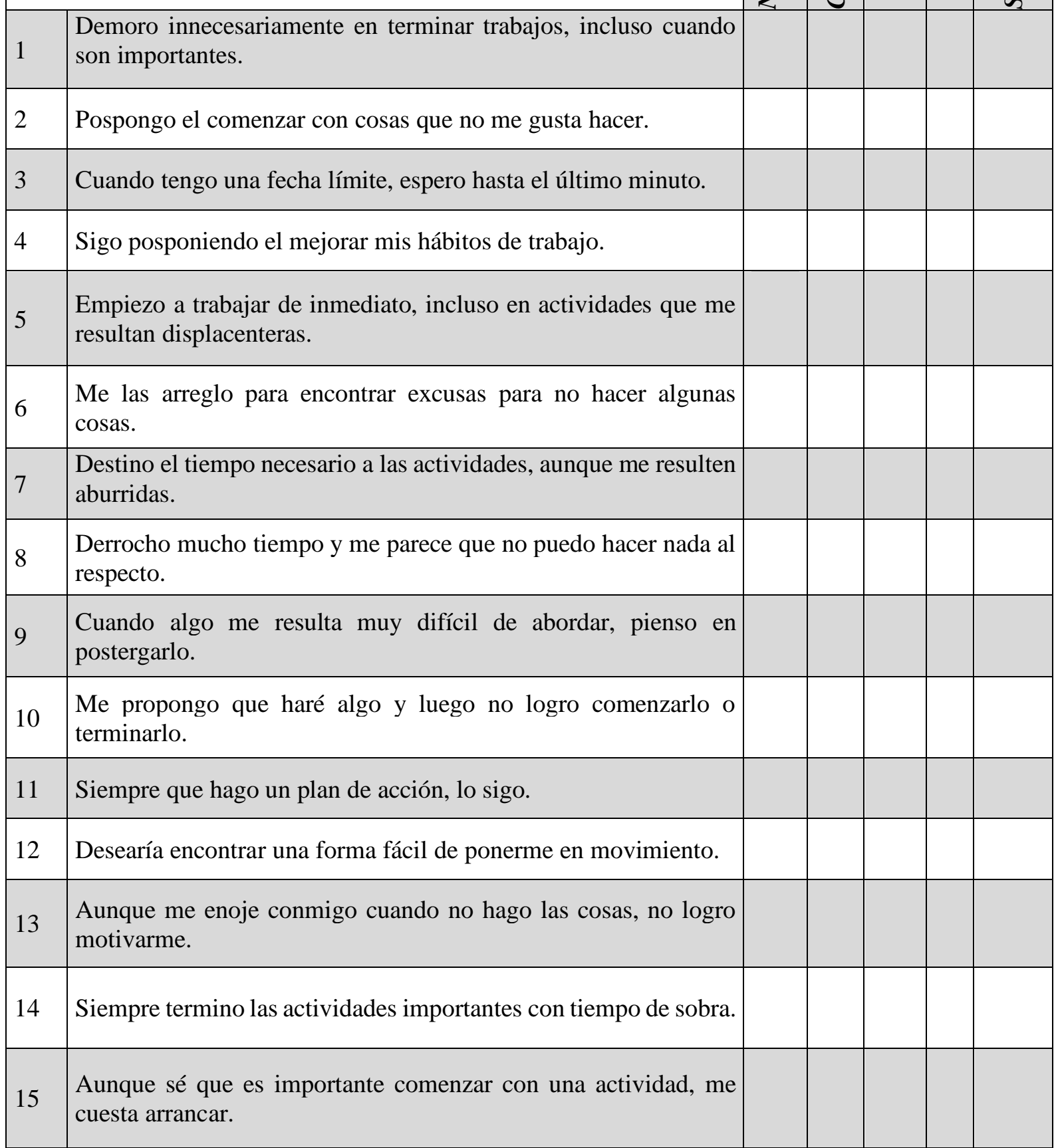

